\title{
Übergänge in die Ausbildung: Welche Rolle spielt die Ausbildungsreife?
}

\author{
Bettina Kohlrausch $\cdot$ Heike Solga
}

Zusammenfassung: Untersuchungen zeigen, dass der Übergang in eine voll qualifizierende Ausbildung für Hauptschüler/innen zunehmend schwieriger wird. Als Erklärung wird häufig die Annahme formuliert, dass mehr und mehr Hauptschulabgänger/innen die erforderliche Ausbildungsreife fehle, d.h. dass sie (noch) nicht den Anforderungen einer beruflichen Ausbildung gerecht würden. Es existieren keine empirischen Studien darüber, ob sich Hauptschulabgänger/innen mit und ohne Ausbildungsplatz wirklich in ihrer Ausbildungsreife unterscheiden. Dieser Frage wird im vorliegenden Beitrag auf Basis von Längsschnittdaten von niedersächsischen Hauptschulabgänger/innen nach Klasse 9 nachgegangen. Zentrale Ergebnisse der Analysen sind: $45 \%$ dieser Abgänger/innen gelingt direkt nach dem Verlassen der Schule der Übergang in eine voll qualifizierende Ausbildung. Dabei spielen fachliche Leistungen eine geringere Rolle als die Note für das Arbeitsverhalten sowie Unterschiede in der betrieblichen Einbindung der Jugendlichen bereits während der Schulzeit. Die Ergebnisse weisen darauf hin, dass vor allem das Sozialverhalten als eine Dimension von Ausbildungsreife sowie - darüber hinausgehend - Möglichkeiten des Entdeckens von Stärken der Jugendlichen und nicht nur ihrer Schwächen wichtige Faktoren für den Erfolg von Hauptschüler/innen beim Übergang in eine Ausbildung sind.

Schlüsselwörter: Ausbildungsreife · Übergangssystem · Ausbildungsmarkt ·

Leistungsschwache Jugendliche · Nicht-kognitive Fähigkeiten · Fachleistungen · Hauptschule

\section{Transitions into apprenticeship in Germany: How important is youth's "maturity for VET"?}

Abstract: Youth with no or only a lower secondary school degree (Hauptschulabschluss) are
increasingly disadvantaged in terms of access to vocational education and training (VET). Their
lower chances of obtaining a trainee are explained by the claim that an increasing number of them
are „not mature enough for VET“. These young people would not (yet) meet the training require-
ments- - so the criticism. So far there are no empirical studies that have shown whether such

Online publiziert: 21.11 .2012

(C) Springer Fachmedien Wiesbaden 2012

Dr. B. Kohlrausch $(\square)$

Soziologisches Forschungsinstitut Göttingen (SOFI)

Friedländer Weg 31, 37085 Göttingen, Deutschland

E-Mail: bettina.kohlrausch@sofi.uni-goettingen.de

Prof. Dr. H. Solga

Abteilung „Ausbildung und Arbeitsmarkt“, Wissenschaftszentrum

Berlin für Sozialforschung (WZB), Reichpietschufer 50, 10785 Berlin, Deutschland

E-Mail: heike.solga@wzb.eu 
immaturity can indeed serve as an appropriate explanation for differences in training chances of less-educated youth. This paper answers this question by analyses using a panel survey of school leavers after grade 9 from the Hauptschule in Lower Saxony. Central results are: About $45 \%$ of the school leavers had successfully entered into an apprenticeship within three months. School grades in German and mathematics were less important than grades for work attitudes and firminternships while still at school. In general, our analyses reveal that social behaviour and a firm's opportunities to discover the strengths of low-achieving youths and not only their weaknesses are important factors for the chances of successful transitions into training.

Keywords: Hauptschule $\cdot$ Low-performing youths · Non-cognitive capabilities · Professionrelated performance $\cdot$ Training maturity $\cdot$ Transition system $\cdot$ Vocational training market

\section{Einleitung}

Empirisch wurde vielfach gezeigt, dass sich der Übergang in eine voll qualifizierende Ausbildung für Hauptschüler/innen - insbesondere dann, wenn sie schlechte Noten haben oder gar die Schule ohne einen Schulabschluss verlassen - zunehmend schwierig gestaltet (s.z.B. Autorengruppe Bildungsberichterstattung 2010; Krekel und Ulrich 2009; Solga 2004, 2005). Im Ausbildungspakt 2009 wird vor allem eine mangelnde Ausbildungsreife bei den ausbildungslosen Jugendlichen (insbesondere den Hauptschüler/innen) und weit weniger das Fehlen von Ausbildungsplätzen als das Kernproblem beim Einmünden in eine voll qualifizierende Ausbildung gesehen (Bundesministerium für Arbeit und Soziales, Pressemitteilung zum Ausbildungspakt 2009).

Empirisch gibt es bisher allerdings keine Untersuchungen dazu, 1) inwieweit Hauptschüler/innen, die sich erfolglos um eine Ausbildung beworben haben, Defizite in ihrer Ausbildungsreife aufweisen und 2) ob sie sich von Hauptschulabgänger/innen mit einem Ausbildungsplatz hierin wirklich unterscheiden. Ein wesentlicher Grund dafür ist, dass es an Daten mangelt, dies zu untersuchen. Mit den Längsschnittdaten aus den Evaluationen der Projekte „Abschlussquote erhöhen - Berufsfähigkeit steigern 1 und 2“ sowie „Vertiefte Berufsorientierung und Praxisbegleitung an Hauptschulen" können diese Fragen systematisch, wenn auch nur für Hauptschulabgänger/innen in Niedersachsen, beantwortet werden.

Kern dieser Projekte war die Einrichtung von Berufsstarter- und Praxisklassen für ,,akut abschlussgefährdete Hauptschüler/innen“ in Niedersachsen. Die Förderung der Schüler/ innen begann in der 8. Klasse und ging bis zum Ende der 9. Klasse. Zentrales Kennzeichen dieser Klassen waren wöchentliche betriebliche Praxistage (in der Regel zwei Unterrichtstage), verbunden mit schulischen Zusatzangeboten zur Berufsorientierung. Mithilfe des betrieblichen Praxisbezugs des Schulalltags sollten die Lern- und Leistungsmotivation sowie die sozialen Kompetenzen der Schüler/innen erhöht und die Ausbildungsplatzsuche unterstützt werden. Diese Klassen wurden zusätzlich von sogenannten Berufsstart- bzw. Praxisbegleiter/innen betreut. Sie sollten den Schüler/innen bei der Suche nach Praktikumsplätzen helfen und sie im Praktikum pädagogisch begleiten. Ähnliche Klassen existieren auch in anderen Bundesländern, z. B. die „Praxisklassen“ in Bayern, die „Schub-Klassen“ in Hessen oder „Betrieb und Schule“ in Nordrhein-Westfalen. 
Die Grundlage für die Analysen des Beitrags bildet die Teilpopulation der Schulabgänger/innen aus den niedersächsischen Berufsstarter- und Praxisklassen sowie aus ihren Parallelklassen, die mit und ohne Hauptschulabschluss die Schule nach Klasse 9 verlassen haben (s. Abschn. 3). Diese Daten sind sehr gut geeignet, die oben formulierten Fragen zu beantworten. Sie umfassen Informationen zu den unterschiedlichen Dimensionen von Ausbildungsreife. Zudem handelt es sich bei diesen Jugendlichen um jene, bei denen besonders große Defizite im Hinblick auf die Ausbildungsreife vorhanden sein sollten. Gegenstand des Beitrags ist nicht die Evaluation dieser Projektklassen und ihres Förderansatzes. Vielmehr sollen die Informationen zu dieser eher leistungsschwachen Gruppe von Hauptschüler/innen dazu dienen, die Bedeutung der Ausbildungsreife für den Erfolg bei der Ausbildungssuche von Hauptschulabgänger/innen zu untersuchen.

Im Folgenden werden zunächst theoretische Überlegungen und anschließend empirische Befunde zu Prozessen vorgestellt, durch die die Defizite in der Ausbildungsreife zu einer Benachteiligung dieser Schulabgängerpopulation führen können. Der Beitrag endet mit einigen Überlegungen zur Bedeutung dieser Befunde für das Konzept der Ausbildungsreife.

\section{Zusammenhang von Ausbildungsreife und Ausbildungschancen}

Es gibt keine einheitliche Definition von Ausbildungsreife (s. Dietrich et al. 2009; Eberhard 2006; Hilke 2004). Verschiedene Akteure (z.B. Wirtschaft und Gewerkschaften) haben unterschiedliche Vorstellungen davon, was unter Ausbildungsreife zu verstehen ist. Während Vertreter der Wirtschaft die wachsende Zahl unvermittelter Ausbildungsbewerber vor allem mit mangelnder Ausbildungsreife erklären, streiten Vertreter der Gewerkschaften in der Regel ab, dass solche Defizite bei Jugendlichen ohne Ausbildungsplatz verstärkt zu finden sind (vgl. Eberhard 2006; Eberhard und Ulrich 2006). Eine wissenschaftliche Definition von Ausbildungsreife ist nicht vorhanden und wahrscheinlich auch nicht sinnvoll, da sie entweder - aufgrund der sehr unterschiedlichen Anforderungen sehr spezifisch auf den jeweiligen Ausbildungsberuf und dessen Anforderungen abgestimmt sein müsste oder, wenn sie allgemein formuliert sein soll, eher als „Versagen in der (konkreten) Ausbildung“ (unter Kontrolle der jeweiligen Ausbildungsbedingungen) zu definieren wäre.

Dementsprechend beziehen wir uns auf die Definition von Ausbildungsreife, die im Kriterienkatalog des „Nationalen Pakts für Ausbildung und Fachkräftenachwuchs Deutschland" formuliert wurde, da sie das Ergebnis eines Verständigungsprozesses wichtiger am Ausbildungsprozess beteiligter Akteure darstellt (Bundesagentur für Arbeit 2009). In diesem Katalog wird Ausbildungsreife gegenüber Berufseignung sowie Vermittelbarkeit abgegrenzt. Sie ist als ein mehrdimensionales Konzept definiert, das fünf Merkmalsbereiche umfasst:

- schulische Basiskenntnisse (z. B. mathematische Grundkenntnisse);

- psychologische Leistungsmerkmale (z.B. logisches Denken oder Bearbeitungsgeschwindigkeit); 
- physische Merkmale ${ }^{1}$ (altersgerechter Entwicklungsstand und gesundheitliche Voraussetzungen);

- psychologische Merkmale des Arbeitsverhaltens und der Persönlichkeit (z. B. Konfliktfähigkeit, Zuverlässigkeit, Leistungsbereitschaft); sowie

- Berufswahlreife (Selbsteinschätzungs- und Informationskompetenz).

Diese Bereiche lassen sich zu drei Dimensionen einer mangelnden Ausbildungsreife zusammenfassen:

1. kognitive Defizite: mangelnde kognitive Voraussetzungen sowie geringe schulische Basiskenntnisse;

2. nicht-kognitive Defizite: a) mangelnde soziale Voraussetzungen (z. B. Arbeitsverhalten, Teamfähigkeit und Umgangsformen) und b) motivationale Defizite;

3. defizitäre Berufswahlreife, d.h. die fehlende Fähigkeit von Jugendlichen, ,ihre eigenen Bedürfnisse und berufsbedeutsamen Fähigkeiten, Fertigkeiten und Kenntnisse (zu kennen) und diese mit wesentlichen Aspekten und Anforderungen von Berufen in Beziehung setzen (zu können)““(Bundesagentur für Arbeit 2009).

Selbst wenn (zunächst) davon ausgegangen wird, dass sich diese Defizite gehäuft bei (leistungsschwächeren) Hauptschulabgänger/innen finden lassen, so ist damit noch nicht die Frage beantwortet, wie diese Defizite bei der Ausbildungsplatzsuche zu Stolpersteinen werden. Zudem handelt es sich bei dem Konzept von Ausbildungsreife um eine Charakterisierung des Angebots an potenziellen Auszubildenden (oder der sogenannten Nachfrage nach Ausbildungsplätzen). Ungeklärt ist hingegen, welche Rolle das jeweilige Ausbildungsplatzangebot für die Ausbildungschancen von Jugendlichen (auch) mit mangelnder Ausbildungsreife spielt. Es gilt daher zu erklären, welche Rolle diese drei Dimensionen der Ausbildungsreife beim Übergang von der Schule in die Ausbildung spielen, d. h. warum sie zu (angebots- und nachfrageseitigen) Benachteiligungen für leistungsschwache Jugendliche auf dem Ausbildungsmarkt werden.

In der Literatur werden vier Grundprozesse genannt, durch die die gering(er)en Übergangschancen leistungsschwacher Jugendlicher verursacht werden (vgl. Solga 2005). Diese sind Verdrängung, statistische Diskriminierung mit diskreditierendem Charakter, soziale Verarmung und Stigmatisierung. Entlang der drei Dimensionen von Ausbildungsreife wird im Folgenden dargestellt, wie sie zu Benachteiligungen auf dem Ausbildungsmarkt führen können.

\section{Dimension 1: Mangelnde kognitive Voraussetzungen sowie geringe schulische Basis-}

kenntnisse. Entsprechend mikroökonomischen Arbeitsmarkttheorien wird der Zugang zu Ausbildungsplätzen durch den Rangplatz in der Bewerberschlange bestimmt, die wiederum durch Leistungsindikatoren der Bewerber/innen als Proxy zukünftiger Produktivität strukturiert ist (Sørensen und Kalleberg 1981; Thurow 1979). Defizitäre kognitive Voraussetzungen sowie geringe(re) schulische Basiskenntnisse (Dimension 1) sollten demzufolge zu einem hinteren Platz in der Bewerberschlange und damit einer ungünstigen Position im Marktwettbewerb um die knappen Ausbildungsplätze führen. Bei einer ungünstigen Relation von Angebot und Nachfrage auf dem Ausbildungsmarkt haben Jugendliche am Ende der Bewerberschlange kaum noch eine Chance, einen Ausbildungsplatz zu erhalten, so dass sie von ehemals oder unter günstigeren Bedingungen erreichba- 
ren Ausbildungsplätzen quasi ausgeschlossen werden (Verdrängung). Ausbildungsreife wäre daher hier eher relativ - im Vergleich zur Leistungsfähigkeit anderer Jugendlicher - und nicht absolut zu verstehen, denn bei einer besseren Angebot-Nachfrage-Relation sollte es diesen Jugendlichen (doch) möglich sein, einen Ausbildungsplatz zu erhalten.

Die Frage ist jedoch, ob Hauptschulabgänger/innen nach Klasse 9 es wirklich (noch) auf die hinteren Plätze der Bewerberschlange schaffen oder ob sie nicht gänzlich aussortiert und damit gar nicht mehr als potenzielle Kandidat/innen wahrgenommen werden. Letzteres, d.h. die Wirkungsweise einer mangelnden Ausbildungsreife als ein absolutes Ausschlusskriterium, das unabhängig von der Angebot-Nachfrage-Relation auf dem Ausbildungsmarkt zu geringen Ausbildungschancen qua Ausschluss vom Bewerberpool führt, kann mit der Signalling-Theorie (Spence 1974; Weiss 1995) erklärt werden. So stellen Bildungszertifikate (wie Schulabschlüsse oder Noten) Marktsignale dar, auf deren Basis in Rekrutierungsprozessen Produktivitätsannahmen getroffen werden. Ein Mechanismus, der Jugendliche mit niedrigen Schulabschlüssen (wie dem Hauptschulabschluss) oder schlechten Noten generell aus der Bewerberschlange ausschließen könnte, ist die statistische Diskriminierung (Arrow 1985) - insbesondere dann, wenn nicht nur von geringe(re)n, sondern zu geringen Fähigkeiten für eine Ausbildung ausgegangen wird.

Allgemein wird von statistischer Diskriminierung dann gesprochen, wenn ,Entscheidungen über das einzelne Individuum auf der Grundlage der Verhaltenswahrscheinlichkeit von Beschäftigungsgruppen“" (Blossfeld und Mayer 1988, S. 265) getroffen werden. Dabei dienen die mit der Gruppenzugehörigkeit verbundenen Stereotype bezüglich des tatsächlichen und möglichen Verhaltens der Gruppenmitglieder als Bewertungskriterien der zukünftigen Leistungsfähigkeit von Individuen: ,[...] groups have expected positions in the labor queue, but individuals do not" (Thurow 1979, S.21). Bei der immer kleiner werdenden Gruppe der Hauptschüler/innen kann erwartet werden, dass bei ihnen - basierend auf der Gruppenzugehörigkeit - von einer immer geringeren Produktivität ausgegangen wird oder, in Bezug auf die Ausbildung, von einer immer geringeren ,trainability“.

Dieses negative Gruppenstereotyp von Hauptschüler/innen könnte in den letzten Jahren von ,geringer“" zu ,zu gering“" umgeschlagen sein, z. B. durch die anhaltenden PISADiskussionen um „Risikoschüler/innen“ und „funktionale Analphabeten“, insbesondere auf Hauptschulen, oder durch den immer kleiner werdenden Anteil an Hauptschüler/ innen und der damit verbundenen Interpretation, diese hätten es trotz Bildungsexpansion nicht geschafft (vgl. Parsons 1959, S. 117; Trow 1977, S. 112 f.). Das Stereotyp „Hauptschüler gleich leistungsschwach“ könnte heute somit so stark diskreditierende Züge angenommen haben, dass diese Hauptschulabgänger/innen nach der 9. Klasse nicht (mehr) am Ende der Bewerberschlange stehen, sondern gänzlich aussortiert werden - und zwar unabhängig von der Angebot-Nachfrage-Relation auf dem Ausbildungsmarkt (Thurow 1975, S. 174).

Ob die Wirkungsweise der ersten Dimension von Ausbildungsreife relativer oder absoluter Natur ist, sollte sich daher daran zeigen, inwieweit Angebot-Nachfrage-Relationen auf dem Ausbildungsmarkt einen Einfluss auf die Ausbildungschancen von Hauptschüler/ innen haben (im Sinne des Verdrängungsmechanismus) oder nicht (als empirische Erwartung für das Vorliegen von statistischer Diskriminierung mit diskreditierenden Zügen). 
Dimension 2a: Mangelnde soziale Voraussetzungen. Produktivitätsannahmen basieren nicht nur auf kognitiven Individualmerkmalen (oder askriptiven Merkmalen, wie Geschlecht oder Migrationshintergrund). In neueren Arbeiten wird hervorgehoben, dass auch nicht-kognitive Fähigkeiten für Produktivitätsannahmen eine Rolle spielen (vgl. z. B. Cappelli 1995; Heckman und Rubinstein 2001). Zum Teil wird sogar argumentiert, dass nicht-kognitive Fähigkeiten (wie Persönlichkeitsmerkmale, Sozialverhalten, Sozialkompetenzen) das wichtigste Rekrutierungskriterium darstellen (Cappelli 1995, S. 109 f.; Rosenbaum und Binder 1997; Heckman und Rubinstein 2001). Gerade bei Hauptschüler/innen, die allgemein als leistungsschwach gelten, könnten Verhaltensmerkmale daher sehr wichtige Auswahlkriterien sein (vgl. Farkas 2003, S. 556). Bezogen auf die Ausbildungsreife-Dimension 2a kann - in ähnlicher Weise wie für Dimension 1 - zwischen einer relativen Wirkungsweise von Defiziten in den nicht-kognitiven Fähigkeiten (Verdrängung) und einer absoluten Wirkungsweise (Diskreditierung) unterschieden werden. Bei Verdrängung führen geringe(re) nicht-kognitive Fähigkeiten zu einem hinteren Platz in der Bewerberschlange, bei diskreditierender statistischer Diskriminierung zu einem Ausschluss aus dem Bewerberpool.

Aus den bisherigen Überlegungen können folgende Hypothesen zur Wirkungsweise der Dimensionen 1 und 2a abgeleitet werden. Mit Blick auf Verdrängungsprozesse wäre zu erwarten:

H1 Je geringer die kognitiven und/oder nicht-kognitiven Fähigkeiten, desto weiter hinten in der Bewerberschlange werden die Jugendlichen platziert und desto geringer sind ihre Chancen, einen Ausbildungsplatz zu erhalten.

H2 Angesichts tendenziell eher geringer kognitiver Fähigkeiten von Hauptschüler/innen könnten soziale Verhaltensmerkmale im Ausbildungsplatzwettbewerb wichtiger sein als kognitive Fähigkeiten.

H3 Die Übergangschancen von Hauptschulabgänger/innen sollten mit der jeweiligen Situation auf dem Ausbildungsmarkt variieren. Abgänger/innen in Arbeitsamtsbezirken mit einer ungünstigen Ausbildungsmarktsituation sollten eine geringere Übergangschance haben als jene in Bezirken mit einer günstigen Situation. ${ }^{2}$

Als Gegenhypothesen dazu können zur statistischen Diskriminierung (mit diskreditierenden Zügen) folgende Erwartungen formuliert werden:

H4 Wenn Personalverantwortliche den Hauptschulbesuch mit fehlender Kompetenz gleichsetzen, so sollten weder die Schulnoten in Deutsch und Mathematik, die kognitiven Grundfähigkeiten noch soziale Verhaltensmerkmale der Hauptschüler/innen einen Einfluss auf ihre Ausbildungschancen haben (Gegenhypothese zu H1 und H2).

H5 Die jeweilige Angebot-Nachfrage-Relation von Ausbildungsplätzen sollte keine Rolle für die Ausbildungschancen von Hauptschüler/innen spielen (Gegenhypothese zu H3).

H6 Betriebliche Praktika könnten allerdings einen „Gegenprozess“ zur statistischen Diskriminierung in Gang setzen, da die Jugendlichen so die Möglichkeit haben, ihre Fähigkeiten unter Beweis zu stellen, so dass ihre individuelle Leistungsfähigkeit (wieder) ins Blickfeld der potenziellen Arbeitgeber/innen geraten kann. Dies sollte ihnen umso eher gelingen, wenn sie (a) Berufsstarter- oder Praxisklassen besucht 
haben (d.h. während der Schulzeit regelmäßig in Betrieben waren) und (b) je länger sie diese Praxistage (während der Schulzeit) im selben Betrieb absolviert haben, d.h. je weniger Betriebswechsel sie bei ihren Praxistagen hatten. Dies würde eine günstige Gelegenheit bieten, eventuelle Vorurteile im persönlichen Kontakt auszuräumen und ein gutes (gegenseitiges) Matching der Interessen der Jugendlichen und eine Akzeptanz im Betrieb herzustellen (in der Literatur wird dies als „Klebeeffekt“ bezeichnet).

Bisher wurden Prozesse des Auswahlverhaltens von Betrieben betrachtet. Motivationale Merkmale der Jugendlichen (Dimension 2b) sowie die Berufswahlreife (Dimension 3) sollten hingegen eher über das Bewerbungsverhalten der Jugendlichen relevant werden.

Dimension 2b: Motivationale Defizite. Auf der Seite der Jugendlichen sind die geringen oder fehlenden Bildungszertifikate häufig mit weiteren negativen Zuschreibungen und Identitätsproblemen verbunden (Solga 2005, S. 158). Solche Stigmatisierungsprozesse können daraus resultieren, dass die Schul- und Bildungskarrieren bildungsschwacher Jugendlicher häufig durch vielfältige Erfahrungen des „Scheiterns“ gekennzeichnet sind (z. B. durch die Zurückstellung bei der Einschulung, das Wiederholen von Klassen oder Schul[typ]wechsel). Diese Erfahrungen erhöhen die Gefahr einer Strategie der Vermeidung erneuter Ablehnung und Erniedrigung - gefolgt von schulischer Desintegration sowie fehlenden Bewerbungsaktivitäten auf dem Ausbildungsmarkt. Daraus resultierende motivationale Defizite (Dimension 2b) können so gesehen durchaus eine rationale Handlungsstrategie zum Erhalt eines hohen Selbstwertgefühls darstellen. In der Konsequenz führt diese Abkühlungsstrategie allerdings zu einem erhöhten Risiko von Ausbildungslosigkeit. Derartige Stigmatisierungsprozesse sind mit quantitativen Analysen schwierig direkt abzubilden. Als indirekte Erwartung für ihre Wirksamkeit können als Hypothesen für Dimension $2 b$ formuliert werden:

H7 Schwänzen als Ausdruck von Schulmüdigkeit (Fernbleiben/Rückzug vom Unterricht) führt zu einer geringeren Bewerbungsaktivität.

H8 Eine geringe Bewerbungsaktivität (bei Kontrolle von Schulleistungen und nicht-kognitiven Merkmalen) könnte eine Rückzugsstrategie darstellen und wäre mit einer geringeren Übergangschance in eine Ausbildung verbunden.

Dimension 3: Defizitäre Berufswahlreife. Ausdruck einer defizitären Berufswahlreife sind mangelnde Kenntnisse über den Berufsalltag und darüber, wie der Weg in die Ausbildung erfolgreich gestaltet werden kann. Ursache für solche Informationsdefizite können Prozesse einer sozialen Verarmung der Umwelt sein. Bei Hauptschüler/innen könnten diese Defizite häufiger auftreten, da sie überdurchschnittlich oft aus Familien stammen, in denen die Eltern keine Berufsausbildung absolviert haben. Mit diesen geringeren kulturellen Ressourcen fehlen hinsichtlich der Ausbildung positive Rollenmodelle, Informationen über Anforderungen und Profile von Berufen sowie das Wissen über das „Wie“ des Sichbewerbens. Fehlende oder defizitäre Bewerbungsstrategien als Ausdruck mangelnder Berufswahlreife (Dimension 3) wären somit auch Folge eines ärmeren familiären Umfeldes (soziale Verarmung) und würden durch mangelhafte Bewerbungsaktivitäten zu 
geringen Ausbildungschancen führen. Daraus kann folgende Hypothese zur Wirkungsweise der 3. Dimension (Berufswahlreife) abgeleitet werden:

H9 Je geringer die kulturellen Ressourcen der Eltern (und damit die Unterstützungspotenziale für die Berufswahl sowie Ausbildungsplatzsuche) sind, desto geringer sollten die Übergangschancen in eine Ausbildung sein.

\section{Daten, Operationalisierung, Methoden}

Zur Prüfung der Hypothesen verwenden wir einen Datensatz, der zur Evaluation der Projekte „Abschlussquote erhöhen - Berufsfähigkeit steigern“ (AQB1 und AQB2) sowie „Vertiefte Berufsorientierung und Praxisbegleitung an Hauptschulen“ (VBOP) erhoben wurde. AQB1 lief vom 2. Halbjahr der 8. Klasse (Februar 2007) bis zum Ende der 9. Klasse (Juli 2008) und AQB2 sowie VBOP vom Beginn der 8. Klasse (Herbst 2008) gleichfalls bis zum Ende der 9. Klasse (Juli 2010). Insgesamt wurden 60 Projektklassen an 46 niedersächsischen Schulen eingerichtet.

Es gab keine klaren Definitionskriterien für die Aufnahme in die Projektklassen. Die Überweisung von Schüler/innen in diese Klassen wurde von den teilnehmenden Schulen sehr unterschiedlich gehandhabt. Wichtig war nur, dass die Lehrer/innen der Auffassung waren, dass diese Schüler/innen voraussichtlich den Hauptschulabschluss oder den Übergang in eine Ausbildung nicht schaffen werden. Bei einem Teil der Schüler/innen wurde dies durch sehr mangelhafte Schulleistungen begründet, bei einem anderen Teil mit Schwänzen und motivationalen Defiziten (auch bei relativ guten Schulleistungen). ${ }^{3}$

Als Kontrollgruppe wurden die Schüler/innen einer Parallelklasse der jeweiligen Projektklasse (an den 46 teilnehmenden Schulstandorten) befragt. Die Entscheidung für dieses Vorgehen der Kontrollgruppenkonstruktion wurde nach Abwägung denkbarer Alternativen aufgrund forschungspraktischer und methodischer Überlegungen getroffen. Ein Feldexperiment konnte nicht durchgeführt werden, da die Schüler/innen für die Berufsstarterklassen bereits bei Auftragserteilung für die Evaluation ausgewählt waren. Bei anderen alternativen Methoden, wie z. B. der Suche nach „statistischen Zwillingen“, wäre die Einbeziehung von mehr als 100 weiteren Schulen erforderlich gewesen, damit eine ausreichende Anzahl „akut abschlussgefährdeter“ Schüler/innen aus „,normalen“ Hauptschulklassen für das Matching verfügbar gewesen wären (zu kostenträchtig).

Mit dem gewählten Design sind ferner unbeobachtete Einflüsse des Schulkontextes, dem beide Klassen angehörten, kontrolliert. Darüber hinaus war das Interesse an der Evaluation der Projekte sehr groß, da die Kontrollklassen auch den Projektschulen angehörten. Dies schlägt sich in hohen Ausschöpfungsquoten - auch für die Kontrollklassen - von ca. 90 \% nieder (s. Solga et al. 2010, S. 11; Solga et al. 2011, S. 14).

Der Nachteil dieser Vorgehensweise ist, dass in der Kontrollgruppe nicht alle Jugendlichen ,statistische Zwillinge“ darstellen. Allerdings werden in diesem Beitrag nur Abgänger/innen aus den Projekt- und den Kontrollklassen berücksichtigt, die bereits nach der 9 . Klasse die Schule verlassen haben. Da es in Niedersachsen auch für Hauptschüler/innen nicht ungewöhnlich ist, die 10. Klasse zu besuchen, ${ }^{4}$ kann davon ausgegangen werden, dass es sich bei diesen Schulabgänger/innen nach der 9. Klasse aus den Kontrollklas- 
sen gleichfalls um eher leistungs- und/oder motivationsschwache Schüler/innen handelt (s. Tab. 1; Solga et al. 2010, S. 59; Solga et al. 2011, S. 65).

Für die Fragestellung des vorliegenden Beitrags ist es darüber hinaus nicht zentral, ob es sich um eine repräsentative Stichprobe von Hauptschulabgänger/innen handelt. Wichtiger ist vielmehr, dass mit den Abgänger/innen von Hauptschulen nach der 9. Klasse eine Schülerpopulation betrachtet wird, die bereits qua Schulabschluss hinsichtlich der ersten Dimension von Ausbildungsreife (schulische Basiskenntnisse) Defizite aufweist. Für unsere Analysepopulation sollte sich daher der negative Einfluss einer mangelnden Ausbildungsreife auf die Ausbildungschancen in besonderer Weise zeigen.

Die Analysen des Beitrags basieren auf Längsschnittdaten, die im Zeitraum von Februar 2007 bis Oktober 2010 durch das Soziologische Forschungsinstitut Göttingen (SOFI) erhoben wurden (vgl. Solga et al. 2010; Solga et al. 2011). In dieser Zeit wurden (fast) alle Schüler/innen der Projekt- und Parallelklassen mehrmals in Klassenrauminterviews während der Schulzeit befragt. Darüber hinaus wurde eine weitere Befragungswelle (mit Telefoninterviews/CATI) drei Monate nach dem Verlassen der Schule durchgeführt.

In unsere Analysen beziehen wir insgesamt 733 Schüler/innen ein, die an den Befragungswellen, in denen die relevanten Informationen erhoben wurden, teilgenommen und die Schule nach der 9. Klasse verlassen haben, sodass sie tatsächlich zur ,,at-risk-population“ für den Übergang in eine Ausbildung gehören. Dabei handelt es sich um 223 Schüler/innen aus den normalen Hauptschulklassen und 510 aus den Projektklassen.

\subsection{Methoden}

Die abhängige Variable ist die Chance des Übergangs in eine voll qualifizierende Ausbildung ( $1=$ Übergang in eine betriebliche, schulische oder überbetriebliche voll qualifizierende Ausbildung; $0=$ kein Übergang). Dementsprechend wurden logistische Regressionen geschätzt. In einem ersten Schritt haben wir - entsprechend unserer Datenstruktur (Schüler/innen in Klassen in Schulen) - eine logistische Mehrebenenregression durchgeführt (mit der STATA-Prozedur xtmelogit). Das Nullmodell zeigte allerdings, dass die Schul- und Klassenebene zusammen nur 3\% der erklärbaren Varianz aufklären können. Daher haben wir einfache binäre logistische Regressionen mit robusten Standardfehlern (mit der wir dennoch die Clusterung der Daten berücksichtigen) geschätzt.

Aufgrund der Skalenverzerrung durch unterschiedliche Varianzanteile verändern sich in logistischen Regressionen die Koeffizienten unabhängiger Variablen, wenn andere Variablen in das Schätzmodell aufgenommen werden - und dies auch dann, wenn diese nicht miteinander korrelieren (s. dazu Mood 2010). Zur Lösung dieses Problems verwenden wir daher nicht Odds Ratios, sondern durchschnittliche Marginaleffekte (average marginal effects, AME). Dieses Vorgehen hat zudem den Vorteil, dass die Koeffizienten (mit 100 multipliziert) als Prozentpunkte der Verringerung/Vergrößerung der Übergangswahrscheinlichkeit in eine Ausbildung interpretiert werden können.

\subsection{Operationalisierung}

In den Hypothesen treten die unterschiedlichen Dimensionen von Ausbildungsreife als unabhängige Variablen auf. Diese sind folgendermaßen definiert. 
Die kognitiven Voraussetzungen der Jugendlichen werden mit dem sprachunabhängigen Subtest „Matrizen“ (zur Erfassung figuraler kognitiver Grundfähigkeiten) aus dem Intelligenz-Struktur-Test 2000R (I-S-T 2000R) abgebildet, welche für Personen ab 15 Jahren einsetzbar sind (vgl. Amthauer et al. 2001). Dabei handelt es sich um eine Dimension der sogenannten fluiden kognitiven Grundfähigkeiten, die deutlich weniger als kristalline kognitive Grundfähigkeiten von den konkreten Lerngelegenheiten abhängig sind (vgl. Cattell 1987; Solga et al. 2005). Der Test wurde zu Beginn des zweiten Halbjahres der 9. Klasse durchgeführt.

Als Indikator der schulischen Basiskenntnisse verwenden wir das Niveau des Hauptschulabschlusses - als Kombination der Mathematik- und Deutschnote der Schüler/innen am Ende der 9. Klasse. Wir unterscheiden zwischen Abgänger/innen mit guten Leistungen (in beiden Fächern die Note $3^{5}$ oder besser), gemischten Leistungen (in einem Fach die Note 3 oder besser, in dem anderen Fach die Note 4 oder schlechter), schlechten Leistungen (in beiden Fächern die Note 4 oder schlechter) und ohne Hauptschulabschluss.

Die sozialen Voraussetzungen der Schüler/innen (Dimension 2a) werden mit der Schulnote im Arbeitsverhalten am Ende der 9. Klasse operationalisiert. Zur Beschreibung der motivationalen Voraussetzungen (Dimension 2b) der Abgänger/innen dienen die Angaben zum mehrmaligen Schwänzen im ersten Halbjahr der 9. Klasse - als Indikator für Schulmüdigkeit - sowie die Anzahl der abgeschickten Bewerbungen bis zum Ende der 9. Klasse.

Zentrale Voraussetzungen für die sogenannte Berufswahlreife sind einerseits das Interesse am Übergang in eine Ausbildung (gemessen über die Frage: „Möchtest du nach der Schule eine Ausbildung beginnen?“) und andererseits die Kenntnis eines gewissen Berufsspektrums, auf deren Grundlage dann eine Entscheidung für den individuellen Wunschberuf getroffen werden kann. Die Jugendlichen wurden daher Mitte der 8. Klasse und der 9. Klasse gefragt, ob sie ihren Wunschberuf benennen können, und am Ende der 9. Klasse, ob sie schon genaue Vorstellungen von ihrem Wunschberuf haben.

Die Ausbildungsmarktsituation wird über die Angebot-Nachfrage-Relation auf dem Ausbildungsmarkt im jeweiligen Arbeitsagenturbezirk des Standorts der Schule entsprechend den Angaben des Berufsbildungsberichts in den Jahren 2007 und 2010 abgebildet. Wir unterscheiden zwischen einer ausgeglichen bis günstigen Situation (mit mehr als 100 Ausbildungsplätzen pro 100 Bewerber/innen) und einer ungünstigen bis sehr ungünstigen (mit weniger als 100 Ausbildungsplätzen pro 100 Bewerber/innen). Obgleich Jugendliche bei der Ausbildungsplatzsuche nicht an die Heimatregion gebunden sind, sondern im gesamten Bundesgebiet eine Ausbildung suchen und aufnehmen können, ist es für die hier betrachtete Abgängerpopulation durchaus berechtigt, im regionalen Heimatkontext (operationalisiert über den Schulstandort) zu verbleiben. Zum einen sind diese Abgänger/innen beim Verlassen der Schule vergleichsweise jung (zwischen 16 und 17 Jahren), sodass die tägliche Mobilität weitgehend an den öffentlichen Nah- und Regionalverkehr gebunden ist. Zum anderen ist die Aufnahme einer Ausbildung außerhalb des Wohnorts mit zusätzlichen Kosten für Unterbringung, Verpflegung und Fahrten verbunden. Diese Kosten schränken die Mobilität ein, da die Jugendlichen großteils aus einkommensschwächeren Familien kommen und zudem, wenn überhaupt, eher in Ausbildungsberufe mit geringen Ausbildungsvergütungen einmünden (wie Verkäufer/in, Lagerist/in, Maler/ in, Lackierer/in). 
Für die Untersuchung des sogenannten Klebeeffekts (H6) wird die Häufigkeit der Wechsel des Praktikumsbetriebs im Projektverlauf verwendet (kein bis zweimaliger, drei- bis fünfmaliger und häufiger als fünfmaliger Wechsel). Diese Angabe ist nur für die Projektschüler/innen vorhanden, da es in den Parallelklassen keine regelmäßigen Praxistage gab.

Indikator der familiären Ressourcen ist der Ausbildungsabschluss der Eltern. Hier unterscheiden wir zwischen Familien, in denen mindestens ein Elternteil über eine berufliche Ausbildung verfügt, und Familien, in denen dies nicht der Fall ist oder in denen die Jugendlichen zu dieser Frage keine Angabe machen können. ${ }^{6}$ Dieser Indikator hat die größte Nähe zum hier untersuchten Ausbildungsübergang und erwies sich zudem im Vergleich zum Erwerbsstatus und zur beruflichen Stellung der Eltern in den Analysen als am erklärungskräftigsten.

Schließlich wird der Migrationshintergrund (Jugendliche, die entweder selbst im Ausland geboren wurden oder mindestens ein Elternteil hatten, welches im Ausland geboren wurde) und das Geschlecht der Jugendlichen kontrolliert. Letzteres ist deshalb wichtig, weil Mädchen etwas bessere Noten erreichen und Jungen etwas häufiger in Projektklassen vertreten sind. Andererseits haben Mädchen, die nach der 9. Klasse die Schule verlassen, den Nachteil, dass ihr Spektrum an Ausbildungsberufen eingeschränkter ist als für Jungen, da viele der frauentypischen Ausbildungsberufe den mittleren Schulabschluss voraussetzen (vgl. Krüger 1991). Damit ist die Konkurrenz unter diesen Schulabgängerinnen um die verfügbaren Ausbildungsberufe größer als bei den Jungen (vgl. Bundesinstitut für Berufsbildung 2010, S.42), sodass Jungen bei gleichen Noten und Abschlüssen höhere Ausbildungschancen haben. Dieser Befund bestätigt sich auch in unseren Analysen (aus Platzgründen haben wir die Effekte der Kontrollvariablen nicht ausgewiesen). Eine Übersicht der Verteilungen und Fallzahlen der abhängigen und unabhängigen Variablen ist in Tab. 1 ausgewiesen.

\section{Deskriptive Befunde zur Ausbildungsreife und Ausbildungschance}

Wie die Informationen in Tab. 1 zeigen, ist die Ausbildungsreife der Hauptschulabgänger/ innen nach Klasse 9 in den Dimensionen 1 sowie $2 \mathrm{a}$ und $2 \mathrm{~b}$ tatsächlich als gering einzuschätzen. Der Hauptschulabschluss ist in der Mehrheit eher schlecht oder gemischt (69 bzw. 69\%), und 7 bzw. 4\% haben die 9. Klasse sogar ohne einen Hauptschulabschluss verlassen. Die kognitiven Grundfähigkeiten bewegen sich in der unteren Hälfte (s. Abb. 1).

Hinsichtlich der motivationalen und sozialen Voraussetzungen fällt die Beurteilung ähnlich aus. Etwa jeder vierte bis fünfte Jugendliche hat im ersten Halbjahr der 9. Klasse die Schule geschwänzt. Ferner erhielt der überwiegende Teil der Schüler/innen für ihr Arbeitsverhalten die Note 3 oder schlechter.

Von einer mangelnden Berufswahlreife (Dimension 3) kann bei den Jugendlichen insbesondere der Projektklassen - hingegen nicht ausgegangen werden. Die überwiegende Mehrheit der Jugendlichen möchte eine Ausbildung machen, hat einen konkreten Berufswunsch und hat sich bereits über die Anforderungen und negativen Seiten ihres Berufswunsches Gedanken gemacht. 
Tab. 1: Operationalisierungen und Deskription. (Quelle: Eigene Berechnungen, at-risk-population (Schüler/innen, die nach der 9. Klasse die Schule verlassen haben), Datensatz „Abschlussquote erhöhen - Berufsfähigkeit steigern 1 und 2“ und „Vertiefte Berufsorientierung und Praxisbegleitung an Hauptschulen", 2007-2010, SOFI)

\begin{tabular}{|c|c|c|c|c|c|}
\hline & PK & NK & PK & NK & $\mathrm{N}$ \\
\hline Abhängige Variablen & & & & & 733 \\
\hline Übergang in eine Ausbildung inner- & & Absolut & 249 & 79 & 328 \\
\hline $\begin{array}{l}\text { halb von drei Monaten nach Verlassen } \\
\text { der Schule }\end{array}$ & & in Prozent & 49 & 35 & 45 \\
\hline
\end{tabular}

\section{Unabhängige Variablen}

Dimensionen von Ausbildungsreife

1) Schulische Basiskenntnisse (in

Spaltenprozenten)

Niveau des Hauptschulabschlusses

Gute Leistungen

Gemischte Leistungen

Schlechte Leistungen

Kein Hauptschulabschluss

Keine Angabe

Beginn 2. Hj. 9. Klasse

Ende 9. Klasse

22,4

22,9

$38,6 \quad 34,1$

$30,8 \quad 35,0$

$7,2 \quad 4,5$

$1,0 \quad 3,5$

2.a) Soziale Voraussetzungen

(in Spaltenprozenten)

Note im Arbeitsverhalten, Ende

9. Klasse

Gut (1-2)

$15,7 \quad 14,8$

Mittel bis schlecht (3-5)

$77,8 \quad 80,2$

Missings

$6,5 \quad 5,0$

2.b) Motivationale Voraussetzungen (in Spaltenprozenten)

Anteil derjenigen, die im 1. Halbjahr Klasse geschwänzt haben

Ja

18,4

23,3

Nein

70,4

67,3

Missings

11,2

9,4

Anzahl abgeschickter Bewer-

bungen bis Ende Klasse 9 (in

Spaltenprozenten)

0

$1-5$

29,6

52,5

6-10

$50,0 \quad 29,6$

Mehr als 10

$9,6 \quad 11,2$

Missings

3) Berufswahlreife

2. Hj. 8. Klasse

$6,7 \quad 4,0$

$4,1 \quad 2,7$

Anteil der Schüler/innen mit Ausbildungsinteresse direkt nach Ende der $81,3 \quad 76,1$

2. Hj. 9. Klasse

$80,4 \quad 66,4$

Schule (in Prozent) 
Tab. 1: (Fortsetzung)

\begin{tabular}{llllll}
\hline & PK & NK & PK & NK & N \\
\hline Anteil der Schüler/innen mit einem & 74,5 & 80,1 & 88,1 & 82,3 &
\end{tabular}

Berufswunsch (in Prozent)

Vorstellung zum Berufswunsch:

Ende 9. Klasse

(1-3; niedrige Werte $=$ hohe $\mathrm{Zu}$ -

stimmung): Hast du schon darüber

nachgedacht, ... (Mittelwerte, kursiv

Standardfehler)

-...wie der Berufsalltag in deinem

Wunschberuf aussehen wird?

-...welche Anforderungen du für diesen Beruf mitbringen musst?

-...was dir an dem Beruf weniger gut gefallen könnte?

Abgeschlossene Berufsausbildung der Eltern (in Prozent)

$\mathrm{Ja}$

47,1

Nein/weiß nicht/keine Angabe

45,7

Missings

7,3

\section{Ausbildungsmarktsituation}

(Arbeitsagenturbezirk Schulstandort, in Prozent)

Günstig/ausgeglichen

Ungünstig/sehr ungünstig

Häufigkeit des Wechsels des Praktikumsbetriebes (zwischen Projektbeginn in Klasse 8 und Ende Klasse 9, in Spaltenprozent)

$\begin{array}{lr}0-2 & 24,9 \\ 3-4 & 43,1 \\ 5 \text { und mehr } & 16,5 \\ \text { Missings } & 15,5\end{array}$

\section{Kontrollvariablen}

Migrationshintergrund

$\mathrm{Ja}$

Nein

Missings

Geschlecht

Mädchen

Junge

Missings

Figurale kognitive Grundfähigkeiten

Gut

Schlecht 
Abb. 1: Verteilung der figuralen kognitiven Grundfähigkeiten von Jugendlichen mit und ohne Übergang in eine Ausbildung drei Monate nach Schulende (Prozentanteile der jeweils erreichten Punktzahl). (Quelle: Eigene Berechnungen, $\mathrm{n}=660$ (at-risk-population $=$ Schüler/innen, die nach der 9. Klasse die Schule verlassen haben), Datensatz „Abschlussquote erhöhen Berufsfähigkeit steigern 1 und 2" und ,Vertiefte Berufsorientierung und Praxisbegleitung an Hauptschulen“, 2007-2010, SOFI)

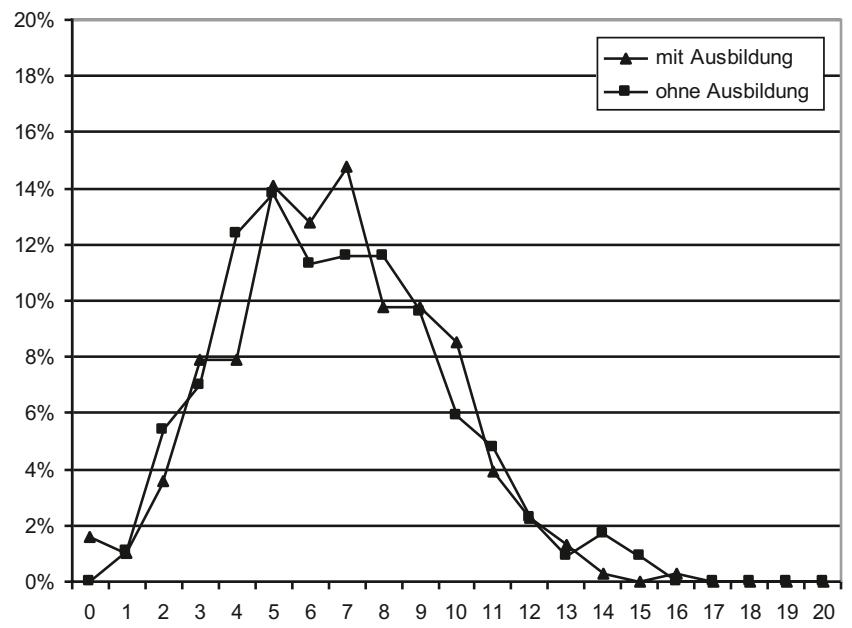

Dreihundertachtundzwanzig der 733 Jugendlichen (45\%) haben drei Monate nach dem Verlassen der 9. Klasse eine voll qualifizierende (betriebliche, schulische oder außerbetriebliche) Ausbildung begonnen. Bei den Abgänger/innen aus den Projektklassen waren es $49 \%$ (249 Jugendliche), bei jenen aus den normalen Hauptschulklassen hingegen nur $35 \%$ (79 Jugendliche).

Ob diese unterschiedliche Erfolgsquote durch den jeweiligen Grad der Ausbildungsreife zu erklären ist, werden die multivariaten Analysen zeigen. Zuvor kann allerdings bereits festgestellt werden, dass sich die figuralen kognitiven Grundfähigkeiten zwischen Jugendlichen mit und ohne Ausbildungsplatz nicht unterscheiden. Sie hatten damit keinen Einfluss auf die Ausbildungschancen der Jugendlichen. Wie Abb. 1 zeigt, ist die Verteilung der in den kognitiven Tests erreichten Punktzahl (abgetragen auf der x-Achse) in beiden Gruppen etwa gleich. In den multivariaten Analysen werden diese Testwerte daher nur noch als Kontrollvariablen berücksichtigt.

\section{Multivariate Analysen zu den Ausbildungschancen}

In den folgenden multivariaten Analysen wird nun untersucht, inwieweit die anderen Faktoren der Ausbildungsreife die Ausbildungschancen der Jugendlichen beeinflusst haben. ${ }^{7}$ Im Modell 1 in Tab. 2 wird deutlich, dass nur die Tatsache, ob ein Hauptschulabschluss erreicht wurde, eine Rolle spielt. Schüler/innen ohne Hauptschulabschluss hatten signifikant schlechtere Chancen, nach der Schule eine Ausbildung zu beginnen. Das Niveau des Hauptschulabschlusses, bezogen auf die Noten in Deutsch und Mathematik, hat hingegen keinen Einfluss. Abgänger/innen mit einem guten Hauptschulabschluss hatten keine signifikant höhere Ausbildungschance als jene mit einem schlechten oder gemischten. Offensichtlich stellt der Hauptschulabschluss eine Mindestvoraussetzung dar, um überhaupt noch eine Chance für einen Ausbildungsplatz zu haben. Damit kann Hypothese 1 in Bezug auf die Noten nicht bestätigt werden. 
Tab. 2: Chance, innerhalb von drei Monaten nach Verlassen der Schule einen Ausbildungsplatz zu finden (Logistische Regressionen, average marginal effects, robuste Standardfehler in Klammern). (Quelle: Eigene Berechnungen, at-risk-population (Schüler/innen, die nach der 9. Klasse die Schule verlassen haben), Datensatz „Abschlussquote erhöhen - Berufsfähigkeit steigern 1 und 2“ und „Vertiefte Berufsorientierung und Praxisbegleitung an Hauptschulen“, 2007-2010, SOFI)

\begin{tabular}{|c|c|c|c|c|}
\hline & M1 & $\begin{array}{l}\text { M2 (nur } \\
\text { Projektschüler) }\end{array}$ & M3 & M4 \\
\hline $\begin{array}{l}\text { Projektklasse } \\
\text { (Nein=Ref.): Ja }\end{array}$ & $0,12 * *(0,04)$ & & $0,09 * *(0,04)$ & $0,13 * *(0,04)$ \\
\hline \multicolumn{5}{|l|}{$\begin{array}{l}\text { Niveau des } \\
\text { Schulabschlusses } \\
\text { (gemischt= Ref.) }\end{array}$} \\
\hline Gut & $0,01(0,05)$ & $-0,02(0,06)$ & $0,01(0,05)$ & $0,00(0,05)$ \\
\hline Schlecht & $-0,03(0,04)$ & $-0,02(0,06)$ & $-0,02(0,04)$ & $-0,03(0,04)$ \\
\hline $\begin{array}{l}\text { Kein Haupt- } \\
\text { schulabschluss }\end{array}$ & $-0,47 * *(0,11)$ & $-0,42 * *(0,11)$ & $-0,41 * *(0,11)$ & $-0,47 * *(0,10)$ \\
\hline \multicolumn{5}{|l|}{$\begin{array}{l}\text { Note im Arbeits- } \\
\text { verhalten (Ende } \\
\text { 9. Klasse) (mittel/ } \\
\text { schlecht=Ref.) }\end{array}$} \\
\hline Gut & $0,17 * *(0,05)$ & $0,12 * *(0,06)$ & $0,17 * *(0,05)$ & $0,17 * *(0,05)$ \\
\hline $\begin{array}{l}\text { ANR (ausge- } \\
\text { glichen=Ref.): } \\
\text { ungünstig/sehr } \\
\text { ungünstig }\end{array}$ & $0,01(0,04)$ & & & \\
\hline \multicolumn{5}{|l|}{$\begin{array}{l}\text { Anzahl der Wech- } \\
\text { sel des Prakti- } \\
\text { kumsbetriebes } \\
(0-2=\text { Ref.) }\end{array}$} \\
\hline $3-4$ & & $-0,09 *(0,05)$ & & \\
\hline 5 und mehr & & $-0,22 * *(0,07)$ & & \\
\hline $\begin{array}{l}\text { Schwänzen im } 1 . \\
\text { Halbjahr Klasse } 9 \\
\text { (nein=Ref.): ja }\end{array}$ & & & $-0,05(0,05)$ & \\
\hline \multicolumn{5}{|l|}{$\begin{array}{l}\text { Beworben bis } \\
\text { Ende Klasse } 9 \\
\text { (nein=Ref.) }\end{array}$} \\
\hline $1-5$ & & & $0,15 * *(0,04)$ & \\
\hline $6-10$ & & & $0,15 * *(0,06)$ & \\
\hline Mehr als 10 & & & $0,09(0,09)$ & \\
\hline
\end{tabular}


Tab. 2: (Fortsetzung)

\begin{tabular}{|c|c|c|c|c|}
\hline & M1 & $\begin{array}{l}\text { M2 (nur } \\
\text { Projektschüler) }\end{array}$ & M3 & M4 \\
\hline \multicolumn{5}{|l|}{$\begin{array}{l}\text { Ausbildungs- } \\
\text { abschluss der } \\
\text { Familie (kein } \\
\text { Elternteil mit } \\
\text { Abschluss = Ref.) }\end{array}$} \\
\hline $\begin{array}{l}\text { Mindestens ein } \\
\text { Elternteil mit } \\
\text { einem Ausbil- } \\
\text { dungsabschluss }\end{array}$ & & & & $0,11 * *(0,03)$ \\
\hline Konstante & $-0,39(0,24)$ & $-0,50 *(0,30)$ & $-0,70 * *(0,27)$ & $-0,59 *(0,25)$ \\
\hline $\begin{array}{l}\text { Anzahl der } \\
\text { Schulabgänger/ } \\
\text { innen }\end{array}$ & 731 & 508 & 731 & 731 \\
\hline Log Likelihood & $-468,47$ & $-328,27$ & $-459,25$ & $-462,12$ \\
\hline $\begin{array}{l}\text { Pseudo-R }{ }^{2} \\
\text { (Nagelkerke) }\end{array}$ & 0,12 & 0,12 & 0,15 & 0,14 \\
\hline
\end{tabular}

Kontrolliert für figurale kognitive Grundfähigkeiten, Geschlecht, Migrationshintergrund, Missing-Kategorien

${ }^{* *} p<0,05, * p<0,1$.

Konform zu Hypothese 2 beeinflusste jedoch die Note im Arbeitsverhalten die Ausbildungschance (und wie die weiteren Modelle zeigen, sehr stabil und unabhängig davon, welche weiteren Variablen aufgenommen wurden). Jugendliche mit einem guten Arbeitsverhalten haben eine um 17 Prozentpunkte höhere Ausbildungswahrscheinlichkeit als Jugendliche mit einem mittelmäßigen oder schlechtem Arbeitsverhalten. Dieser Befund spricht für Hypothese 2 und führt zur Ablehnung von Hypothese 4, mit der erwartet wurde, dass aufgrund von Diskreditierungsprozessen gegenüber Hauptschüler/innen weder die kognitiven noch die nicht-kognitiven Merkmale die Ausbildungschance beeinflussen.

Andererseits zeigen die nicht signifikanten Effekte für die Angebot-Nachfrage-Relation (ANR) auf dem Ausbildungsmarkt in Modell 1, dass Verdrängungsprozesse nur eingeschränkt am Werk sind. Die Ausbildungsmarktsituation hat keinen signifikanten Einfluss auf die Ausbildungschance der Hauptschulabgänger/innen. Damit kann Hypothese 3 nicht, aber Hypothese 5 bestätigt werden. Dieser Befund, dass regionale Standortfaktoren keinen Einfluss auf die Ausbildungschancen von Jugendlichen mit Hauptschulabschluss haben, wird durch Analysen von Kleinert und Jacob (2012) unterstützt. In ihren kohortenvergleichenden Analysen auf Basis einer für Deutschland repräsentativen Studie (ALWA) konnten sie zeigen, dass in der Schulabgängerkohorte 1995-2005 für Hauptschüler/innen diese keinen Einfluss mehr haben - dies jedoch in früheren Kohorten der Fall gewesen ist und auch heute noch für Abgänger/innen mit Realschulabschluss oder Abitur zutrifft.

Des Weiteren gibt es in den Modellen M1, M3 und M4 konform zu Hypothese H6a einen positiven Einfluss des Besuchs einer Projektklasse auf die Ausbildungschancen 
der Jugendlichen. Zudem zeigen die signifikanten Effekte für den Praktikumswechsel in Modell 2 - wie in Hypothese H6b erwartet -, dass häufigere Wechsel die Ausbildungschance deutlich verringert haben, oder umgekehrt, dass durch eine längere Verweildauer im Praktikumsbetrieb die Ausbildungschance (und damit die Sichtbarkeit individueller Fähigkeiten) erhöht werden konnte. Jugendliche, die den Praktikumsbetrieb drei- bis viermal gewechselt haben, hatten eine um 9 Prozentpunkte geringere Ausbildungschance als Jugendliche mit maximal zwei Wechseln; Jugendliche mit fünf und mehr Wechseln eine um 22 Prozentpunkte geringere. Von den Jugendlichen der Berufsstarterklassen, die eine betriebliche Ausbildung begonnen haben $(n=216)$, sind 186 Jugendliche $(86 \%)$ in ihrem Praktikumsbetrieb verblieben. Nur 30 Jugendliche (14\%) haben in einem anderen Betrieb eine Ausbildung begonnen.

Da in Modell 2 für das Arbeitsverhalten kontrolliert wurde, kann der positive Einfluss einer längeren Verweildauer im Praktikum nicht primär mit vorteilhaften Verhaltenseigenschaften erklärt werden. Vielmehr muss nach Erklärungen gesucht werden, die darüber hinausgehen. Eine plausible Möglichkeit ist, dass die Praxistage als gegenseitiges Screening für Jugendliche wie Betriebe fungierten. Die Absolvierung der Praxistage über eine längere Dauer im selben Betrieb erhöhte die Qualität von Matching-Prozessen zwischen Betrieb und Jugendlichen (oder die Möglichkeit von sogenannten Klebeeffekten). Sie vergrößerte bei den Praktikumsbetrieben die Bereitschaft, diesen Jugendlichen eine Chance zu geben, und bei den Jugendlichen die Chance, ,ihren“ Betrieb, in dem sie akzeptiert und gefördert werden, zu finden. Damit kann Hypothese 6 insgesamt bestätigt werden.

Bewerbungsaktivitäten hatten - wie mit Hypothese 8 erwartet - einen positiven signifikanten Einfluss auf die Ausbildungschance (Modell 3). Das Schwänzen als Indikator für Schulmüdigkeit hatte hingegen - anders als in Hypothese 7 angenommen - keinen eigenständigen signifikanten Einfluss auf die Ausbildungschancen. Letzteres könnte der signifikanten Korrelation mit der Note im Arbeitsverhalten $(r=0,15)$ geschuldet sein.

Damit kommen wir zur Dimension 3, der Berufswahlreife. Der Effekt des Bildungshintergrunds der Eltern (als wichtiger Aspekt der kulturellen Ressourcen des Elternhauses) ist signifikant (Modell 4). Jugendliche, die in Elternhäusern aufwachsen, in denen mindestens ein Elternteil eine abgeschlossene Berufsausbildung hat, haben eine um durchschnittlich 11 Prozentpunkte höhere Ausbildungswahrscheinlichkeit als jene aus Familien, in denen kein Elternteil eine abgeschlossene Berufsausbildung hat. Zudem zeigt sich, dass Jugendliche, die mindestens einen Elternteil mit einer abgeschlossenen Berufsausbildung haben, signifikant häufiger darüber nachgedacht haben, wie der Berufsalltag in ihrem Wunschberuf aussehen wird $(r=0,11)$, welche Anforderungen sie für diesen Beruf mitbringen müssen $(r=0,10)$ und was ihnen an dem Beruf weniger gut gefallen könnte $(r=0,18)$. Somit kann Hypothese 9 zu Hindernissen für die Berufswahlreife bestätigt werden.

\section{Fazit}

Ausgangspunkt des Artikels waren die Fragen, ob die Defizitdiagnosen einer fehlenden Ausbildungsreife tatsächlich auf Hauptschulabgänger/innen (hier nach der 9. Klasse) 
zutreffen und, wenn ja, ob sie die Übergangschance für eine berufliche Ausbildung beeinflussen. Zunächst ist festzustellen, dass Defizite teilweise bei den untersuchten Jugendlichen vorhanden sind - allerdings nur in den ersten beiden Dimensionen: mangelnde kognitive Voraussetzungen und schulische Basiskenntnisse sowie mangelnde motivationale und soziale Voraussetzungen. Hinsichtlich der dritten Dimension, der Berufswahlreife, zeigen sich hingegen keine Defizite. So hat der überwiegende Teil der Hauptschüler/ innen konkrete Berufswünsche und das klare Ziel, unmittelbar nach der Schule eine Berufsausbildung zu beginnen. Zudem haben sich auch viele von ihnen Gedanken über den konkreten Berufsalltag gemacht und durchaus realistische Berufswünsche, wie Verkäufer/in, Friseur/in oder Maler/in und Lackierer/in, entwickelt (vgl. Solga et al. 2010, 2011).

Damit stellt sich die Frage, ob sich die Probleme von Hauptschüler/innen beim Übergang in eine berufliche Ausbildung durch die vorhandenen Defizite in der ersten und zweiten Dimension von Ausbildungsreife erklären lassen. Die Ergebnisse unserer Analysen zeigen zum einen, dass mit dem Konzept der Ausbildungsreife die Bedeutung von schulischen Basiskenntnissen und kognitiven Fähigkeiten überschätzt wird (Dimension 1). So haben zwar die Abgänger/innen mit ihrem (einfachen) Hauptschulabschluss nach Klasse 9 per Definition einen vergleichsweise niedrigen Schulabschluss, und auch die Ergebnisse der kognitiven Tests waren sehr niedrig, dennoch gelang es $45 \%$ der Jugendlichen, innerhalb von drei Monaten nach der 9. Klasse einen Ausbildungsplatz zu finden. Lag ein Hauptschulabschluss vor, so konnten die Fachnoten in Deutsch und Mathematik nicht erklären, wer einen Ausbildungsplatz bekommen hat und wer nicht. Auch die Ausbildungsmarktsituation spielte keine Rolle. Wichtiger waren vielmehr die Note im Arbeitsverhalten (Dimension 2) sowie die Praxistage im Betrieb (insbesondere die Verweildauer im selben Betrieb).

Wie sind die Befunde des besonderen Einflusses des Arbeitsverhaltens sowie der besonderen Bedeutung des Praktikums zu erklären? In diesem Beitrag wurde nicht nur untersucht, welche Defizite der Jugendlichen eine Rolle spielen, sondern auch, wie diese im Übergangsprozess wirksam werden. Dabei wurde deutlich, dass weniger Verdrängungs-, sondern vielmehr Diskreditierungs- bzw. statistische Diskriminierungsprozesse den Übergang von Hauptschüler/innen in eine berufliche Ausbildung strukturieren. Das heißt, die geringen Schulleistungen der Hauptschüler/innen sind weniger ein relatives, als vielmehr ein absolutes Ausschlusskriterium. Somit wird sich ihre Situation auch nicht (wieder) verändern, wenn es zukünftig weniger Ausbildungsplatzsuchende gibt. Die Angebot-Nachfrage-Situation auf dem Ausbildungsmarkt spielt für Hauptschulabgänger/ innen nach der 9. Klasse kaum eine Rolle. Dies deutet darauf hin, dass die Erwartungen der Arbeitgeberseite hinsichtlich der schulischen Basiskenntnisse von Abgänger/innen der 9. Klasse inzwischen so gering zu sein scheinen, dass sie das Einstellungsverhalten nur wenig beeinflussen. Eher scheint die Note für das Arbeitsverhalten den Betrieben als ein wesentlicher Ausweis für „Ausbildungsfähigkeit“ zu dienen. Dafür spricht auch die Tatsache, dass Jugendliche, die eine Projektklasse mit betrieblichen Praxistagen besucht haben und die länger im Praktikumsbetrieb verblieben sind, eine höhere Ausbildungschance hatten. Sie hatten die Möglichkeit, im Betrieb ihre Fähigkeiten unter Beweis zu stellen und damit Diskreditierungsprozessen entgegenzuwirken. Sie wurden wieder mit ihren individuellen Fähigkeiten und Motivationen von den Betrieben wahrgenommen 
und konnten dadurch, ,ihre“ Betriebe finden. Und Betriebe hatten wiederum die Möglichkeit, ,ihre“ Jugendlichen zu finden.

Bildungs- und arbeitsmarktpolitisch weisen diese Befunde darauf hin, dass aufgrund der starken Vorbehalte gegenüber Hauptschüler/innen Maßnahmen, die einen direkten Weg in eine Ausbildung fördern, deutlich effektiver sind als indirekte Maßnahmen, die auf eine Verbesserung der Ausbildungsreife zielen. Hinsichtlich der derzeitigen Diskussion zum Fachkräftemangel und zur demografischen Entwicklung legen die Befunde nahe, dass sich der schwierige Zugang zu Ausbildung für Hauptschüler/innen (oder leistungsschwache Schüler/innen) ,demografisch“ nicht von selbst lösen wird. Eine Entspannung der Angebots-Nachfrage-Relation auf dem Ausbildungsmarkt allein kann wohl die Situation dieser Jugendlichen kaum verbessern (s. dazu auch Kleinert und Jacob 2012).

Zudem zeigt sich, dass „Ausbildungsreife“ - anders als es die im Ausbildungspakt vorgenommenen Definitionen nahelegen - kein klar definiertes Bündel von Kompetenzen und Fähigkeiten ist, die vor einer Ausbildung vorhanden sein müssen, damit der Übergang in eine Ausbildung gelingt. Wäre dies der Fall, dann hätten deutlich weniger der untersuchten Hauptschulabgänger/innen in Niedersachsen angesichts der Defizite gerade im schulischen und kognitiven Bereich eine Ausbildungsstelle finden dürfen, als dies real der Fall gewesen ist.

\section{Anmerkungen}

1 Physische Merkmale könne im Folgenden nicht betrachten werden, da dazu keine Informationen vorliegen.

2 Da die Projektklassen die Ausbildungsmarktsituation nicht verändern, sollten sich - bei Kontrolle individueller Merkmale - keine Unterschiede zwischen Abgänger/innen aus Projekt- und „,normalen“ Hauptschulklassen zeigen.

3 Neunundzwanzig Prozent der Teilnehmer/innen der drei Projekte haben die Projektklassen vorzeitig verlassen. Sie sind nicht Teil der Untersuchungspopulation dieses Beitrags. Darunter waren v. a. Schüler/innen, die der Schule mehrfach und dauerhaft ferngeblieben sind oder starke Verhaltensauffälligkeiten (oder gar Gewaltverhalten) zeigten. Berufsstarter- und Praxisklassen sind somit keine Projekte für Schulverweigerer. Die Nichtberücksichtigung der Abbrecher beeinflusst unsere Untersuchung insofern, als Stigmatisierungsprozesse (der Rückzug aus Schule und Leistungskontexten) und motivationale Defizite (Dimension 2b) in den Analysen unterschätzt werden.

4 Werden alle im Projekt befragten Schüler/innen betrachtet, so haben ca. $90 \%$ der Projekt- und knapp 30\% der Kontrollschüler/innen die Schule nach der 9. Klasse verlassen. Die anderen befragten Jugendlichen besuchten jeweils die 10. Klasse.

5 Die Note 3 wurde mit Blick auf den Mittelwert der Noten dieser Schülerpopulation als Unterscheidungskriterium gewählt. Die Abgänger/innen mit guten Leistungen sind damit besser als der Durchschnitt. 
6 Mehr als ein Drittel der Schüler/innen der Projekt- sowie der normalen Klassen haben zu dieser Frage - trotz Erläuterung - keine Angabe gemacht/machen können. Dieser hohe Anteil ist üblich für diese Bildungsgruppe in Befragungen. Er kann zweierlei bedeuten: Entweder wissen diese Schüler/innen nicht, welche Ausbildung ihre Eltern absolviert haben, oder es ist ihnen unangenehm anzugeben, dass ihre Eltern keine Berufsausbildung abgeschlossen haben. In den Analysen werden Schüler/innen, die zu dieser Frage keine Angabe gemacht haben, daher mit den Schüler/innen, die angaben, dass ihre Eltern keine abgeschlossene Berufsausbildung haben, gemeinsam einer Kategorie zugeordnet.

7 Für die Hypothesen 1 bis 4 wäre eine Prüfung des Einflusses der einzelnen Prädiktoren nicht adä-quat. Vielmehr geht es um deren jeweilige Wirkungsweise unter Berücksichtung der jeweils in den anderen drei Hypothesen genannten Faktoren (als Ceteris-paribus-Bedingungen). Daher wurden die in Modell 1 integrierten unabhängigen Variablen nicht schrittweise eingeführt.

\section{Literatur}

Amthauer, R., Brocke, B., Liepmann, D., \& Beauducel, A. (2001). I-S-T 2000R. Intelligenz-Struktur-Test 2000R. Göttingen: Hogrefe.

Arrow, K. J. (1985). Models of discrimination. In K. J. Arrow (Hrsg.), Collected papers of Kenneth J. Arrow (S. 89-111). Cambridge: Harvard University Press.

Autorengruppe Bildungsberichterstattung. (2010). Bildung in Deutschland 2010. Bielefeld: W. Bertelsmann.

Blossfeld, H.-P., \& Mayer, K. U. (1988). Arbeitsmarktsegmentation in der Bundesrepublik Deutschland. Kölner Zeitschrift für Soziologie und Sozialpsychologie, 40(2), 262-283.

Bundesagentur für Arbeit. (2009). Kriterienkatalog zur Ausbildungsreife. Nationaler Pakt für Ausbildung und Fachkräftenachwuchs in Deutschland. http://www.arbeitsagentur.de/zentraler-Content/Veroeffentlichungen/Ausbildung/Kriterienkatalog-zur-Ausbildungsreife.pdf. Zugegriffen: 20. Feb. 2012.

Bundesinstitut für Berufsbildung. (2010). Datenreport zum Berufsbildungsbericht 2010. Bielefeld: W. Bertelsmann.

Bundesministerium für Arbeit und Soziales. (13.10.2009). Ausbildungspakt auch im Krisenjahr 2009 erfolgreich. Pressemitteilung. Berlin: BMAS.

Cappelli, P. (1995). Is the ,skills gap“ really about attitudes? California Management Review, 37, $108-124$.

Cattell, R. B. (1987). Intelligence: Its structure, growth, and action. New York: Elsevier.

Dietrich, H., Dressel, K., Janik, F., \& Ludwig-Mayerhofer, W. (2009). Ausbildung im dualen System und Maßnahmen der Berufsvorbereitung. In J. Möller \& U. Walwei (Hrsg.), Handbuch Arbeitsmarkt 2009 (S. 317-357). Bielefeld: W. Bertelsmann.

Eberhard, V. (2006). Das Konzept der Ausbildungsreife - ein ungeklärtes Konstrukt im Spannungsfeld unterschiedlicher Interessen. Schriftenreihe des Bundesinstituts für Berufsbildung. Bonn: Bundesinstitut für Berufsbildung.

Eberhard, V., \& Ulrich, G. (2006). Schulische Vorbereitung und Ausbildungsreife. In V. Eberhard, A. Krewerth, \& G. Ulrich (Hrsg.), Mangelware Lehrstelle. Zur aktuellen Lage auf der Ausbildungsplatzbewerber in Deutschland (S.35-56). Bielefeld: W. Bertelsmann.

Farkas, G. (2003). Cognitive skills and noncognitive traits and behaviors in stratification processes. Annual Review of Sociology, 29, 541-562.

Heckman, J. J., \& Rubinstein, Y. (2001). The importance of noncognitive skills. The American Economic Review, 91, 145-149. 
Hilke, R. (2004). Beitrag des Referenten Prof. Reinhard Hilke, Psychologischer Dienst der Bundesagentur für Arbeit. In KAUSA (Hrsg.), Fachtagung: Fit für die Arbeit - Können, was Zukunft hat (S. 99-104). Bielefeld: W. Bertelsmann.

Kleinert, C., \& Jacob, M. (2012, im Erscheinen). Strukturwandel des Übergangs in eine berufliche Ausbildung. In R. Becker \& H. Solga (Hrsg.), Soziologische Bildungsforschung (Sonderband 52 der Kölner Zeitschrift für Soziologie und Sozialpsychologie). Wiesbaden: VS Verlag für Sozialwissenschaften.

Krekel, E. M., \& Ulrich, J. G. (2009). Jugendliche ohne Berufsabschluss. Handlungsempfehlungen für die berufliche Bildung. Berlin: Friedrich-Ebert-Stiftung.

Krüger, H. (1991). Doing Gender. Geschlecht als Statuszuweisung im Berufsbildungssystem. In D. Brock, B. Hantsche, G. Kühnlein, \& H. Meulemann (Hrsg.), Übergänge in den Beruf (S. 139169). München: Verlag Deutsches Jugendinstitut.

Mood, C. (2010). Logistic Regression: Why we cannot do what we think we can do, and what we can do about it. European Sociological Review, 26(1), 67-82.

Parsons, T. (1959). The school class as a social system. Harvard Educational Review, 29(Fall), 297-318.

Rosenbaum, J. A., \& Binder, A. (1997). Do employers really need more educated youth? Sociology of Education, 70, 68-85.

Solga, H. (2004). Ausgrenzungserfahrungen trotz Integration - Die Übergangsbiografien von Jugendlichen ohne Schulabschluss. In S. Hillmert \& K. U. Mayer (Hrsg.), Geboren 1964 und 1971 - Neuere Untersuchungen zu Ausbildungs- und Berufschancen in der Bundesrepublik Deutschland (S.39-63). Wiesbaden: VS Verlag für Sozialwissenschaften.

Solga, H. (2005). Ohne Abschluss in die Bildungsgesellschaft. Opladen: Barbara Budrich.

Solga, H., Stern, E., Rosenbladt, B. v., Schupp, J., \& Wagner, G. G. (2005). Measuring learning potentials in survey studies. The case of teenagers in the German Socio-Economic Panel Study (SOEP) (DIW Research Notes 10/2005). Berlin: DIW.

Solga, H., Kohlrausch, B., Kretschmann, C., \& Fromm, S. (2010). Evaluation des Projekts „Abschlussquote erhöhen - Berufsfähigkeit steigern" (IAB-Forschungsbericht 5/2010). Nürnberg: IAB.

Solga, H., Baas, M., \& Kohlrausch, B. (2011). Übergangschancen benachteiligter Hauptschülerund Hauptschülerinnen. Evaluation der Projekte „Abschlussquote erhöhen - Berufsfähigkeitsteigern 2“ und „Vertiefte Berufsorientierung“ (IAB-Forschungsbericht 6/2011). Nürnberg: IAB.

Sørensen, A. B., \& Kalleberg, A. L. (1981). An outline of a theory of matching persons to jobs. In I. Berg (Hrsg.), Sociological perspectives on labor markets (S.49-74). New York: Academic Press.

Spence, M. (1974). Market signalling: Informational transfer in hiring and related screening processes. Cambridge: Harvard University Press.

Thurow, L. C. (1975). Generating inequality. New York: Basic Books.

Thurow, L. C. (1979). A job competition model. In M. J. Piore (Hrsg.), Unemployment and inflation. New York: M. E. Sharpe.

Trow, M. (1977). The second transformation of American secondary education. In J. Karabel \& A. H. Halsey (Hrsg.), Power and ideology in education (S. 105-118). New York: Oxford University Press.

Weiss, A. (1995). Human capital vs. signalling explanations of wages. Journal of Economic Perspectives, 9, 133-154. 